Proceedings of the International Symposium

Capture Gamma-ray Spectroscopy and Related Topics

Santa Fe, New Mexico, August 29-September 3, 1999

\title{
Electromagnetic response of light nuclei
}

\author{
Alberto Mengoni*th and Takaharu Otsuka ${ }^{\dagger h}$ \\ *ENEA, Applied Physics Division, Via Don Fiammelli 2 - 40129 Bologna, Italy \\ ${ }^{\dagger}$ The University of Tokyo, Department of Physics, 2-3-5 Hongo, Tokyo 113, Japan \\ ${ }^{\natural}$ RIKEN, 2-1 Hirosawa, Wako, Saitama 351-01, Japan
}

\begin{abstract}
We show examples of neutron capture, photo dissociation and Coulomb dissociation processes, relevant for studying nuclear structure properties of some light nuclei. In the case of the neutron capture, we show how interference effects among the direct and resonance processes can be accounted for. The same interference effect is shown to play an important role in the photo dissociation of ${ }^{9} \mathrm{Be}$, for excitation energies just above the neutron emission threshold. Finally, the Coulomb dissociation of radioactive projectiles is shown to provides basic structure information on neutron rich exotic systems. The example of the ${ }^{19} \mathrm{C}$ halo structure is shown.
\end{abstract}

\section{INTRODUCTION}

Nuclear structure properties of light nuclei can be investigated by studying their response to electromagnetic excitations. One way to adopt this method is the traditional slow and/or fast neutron capture $\gamma$-ray emission spectroscopy. In addition, textbook examples of photo-dissociation processes (e.g. $\gamma+d \rightarrow n+p$ ) exist showing how the time-reversal reaction channel can also be a corresponding powerful technique. Coulomb excitation processes have been traditionally employed to study the structure properties of nuclear bound states. More recently, a technique has been developed in which the Coulomb excitation process has been extended above the dissociation limit (namely above some nuclear breakup channel). The use of this technique became particularly attractive with the availability of radioactive ion beams (RIBs). In fact, using RIBs this method allows to do spectroscopy of nuclei far from the stability line, up to the drip-lines. We will present here examples of each one of these techniques just mentioned, with particular emphasis on new developments concerning the traditional approaches as well as with some very recent results obtained with the up-to-date RIBs facilities. 


\section{NEUTRON CAPTURE}

For light nuclei, the neutron capture process is often dominated by a direct radiative capture process (DRC). This is a process in which the incident neutron makes a direct transition into one of the bound states of the residual nucleus emitting one or more $\gamma$-rays, without forming any intermediate compound state. DRC models have been formulated in different ways, but with, of course, the same physical contents. To carry on the discussion on the neutron capture as well as on the dissociation processes we need to give here one of these formulation. We start from the wave functions $\Psi_{c}$ and $\Phi_{b}$ of the continuum and of the bound-state, respectively given by

$$
\Psi_{c}(r)=\sum_{l_{c} j_{c}} i^{l_{c}} \frac{\psi_{E_{c} l_{c} j_{c}}(r)}{r}\left[\left[\hat{Y}_{l_{c}} \times \hat{\chi}_{s}\right]^{j_{c}} \times \hat{\chi}_{I}\right]^{J_{c}}
$$

and

$$
\Phi_{b}(r)=\sum_{l_{b} j_{b}} B_{j_{b}, J_{b} \alpha_{b}, I \alpha} \frac{\phi_{n_{b} l_{b} j_{b}}(r)}{r}\left[\left[\hat{Y}_{l_{b}} \times \hat{\chi}_{s}\right]^{j_{b}} \times \hat{\chi}_{I}\right]^{J_{b}}
$$

Here, $\hat{Y}_{l}$ and $\hat{\chi}_{s}$ are angular and spin components of the wave functions, the spin of the target nucleus is indicated by $I$ and all other quantum numbers defining the continuum, bound and core state uniquely are indicated by $\alpha_{c}, \alpha_{b}$ and $\alpha$ respectively. The $B$ 's coefficients are the fractional parentage amplitudes determined by the total number of particles occupying the $n_{b} l_{b} j_{b}$ orbit, $N$. In shell-model standard notation [1] they are indicated by $\left.<\left(j_{b}\right)^{N} J_{b} \alpha_{b} \mid\right\}\left(j_{b}\right)^{N-1} I \alpha>$ and the spectroscopic factor for a given configuration is simply given by $S=N \cdot B^{2}$. The radial wave functions $\psi_{E_{c} l_{c} j_{c}}(r)$ and $\phi_{n_{b} l_{b} j_{b}}(r)$ are obtained by solving the scattering and the bound state problem respectively, in a given potential. The matrix elements for

electric $L$-pole transitions are $Q_{c \rightarrow b}^{(\mathrm{EL})}=<\Psi_{c}\left\|\hat{T}^{\mathrm{EL}}\right\| \Phi_{b}>$ and may be written as a product of three terms

$$
Q_{c \rightarrow b}^{(\mathrm{EL})}=\mathcal{I}_{c b} \cdot B_{b} \cdot A_{c b}
$$

with the electric transition operator $\hat{T}_{M}^{\mathrm{EL}}=r^{L} Y_{L M}$ and the radial overlap integral defined as

$$
\mathcal{I}_{c b}=\int \psi_{E_{c} l_{c} j_{c}}^{*}(r) r^{L} \phi_{n_{b} l_{b} j_{b}}(r) d r
$$

The angular-spin coupling coefficient is indicated by $A_{c b}$ and its definition can be found in the reference [2]. The capture cross section is given by

$$
\sigma_{c \rightarrow b}^{(\mathrm{EL})}=\frac{8 \pi(L+1)}{L[(2 L+1) ! !]^{2}} \frac{k_{\gamma}^{2 L+1}}{\hbar v} \frac{1}{2 s+1} \frac{1}{2 I+1} \bar{e}_{\mathrm{EL}}^{2} \sum_{l_{c} j_{c}, l_{b} j_{b}}\left|Q_{c \rightarrow b}^{(\mathrm{EL})}\right|^{2}
$$


where the sum is extended to all components of the the continuum and bound state wave functions compatible with the EL selection rules. Here, $k_{\gamma}=\epsilon_{\gamma} / \hbar c$ is the photon wave number corresponding to a transition energy $\epsilon_{\gamma}$ and $v$ the coreneutron relative velocity in the continuum. $\bar{e}_{\mathrm{EL}}$ is the single-particle effective charge of the neutron.

In the presence of a resonance state in the vicinity of the continuum energy $E_{c}$, the capture cross section contains a Breit-Wigner and an interference terms, in addition to the DRC term just shown

$$
\sigma(E)=\sigma_{D R C}(E)+\sigma_{B W}(E)+2\left[\sigma_{D R C}(E)+\sigma_{B W}(E)\right]^{1 / 2} \cos \left[\delta_{r}(E)\right]
$$

with

$$
\sigma_{B W}(E)=\pi \lambda_{n}^{2} g_{J} \frac{\Gamma_{n} \Gamma_{\gamma}}{\left(E_{r}-E\right)^{2}+\frac{1}{4} \Gamma^{2}}
$$

and

$$
\delta_{r}(E)=\tan ^{-1}\left[\frac{\Gamma(E)}{2\left(E-E_{r}\right)}\right] .
$$

Here, the resonance energy, the neutron, gamma and total widths are indicated by $E_{r}, \Gamma_{n}, \Gamma_{\gamma}$ and $\Gamma$, respectively. The statistical weight factor is as usual $g_{J}=$ $\left(2 J_{b}+1\right) /(2 I+1)(2 s+1)$. The $\Gamma_{\gamma}$ is intended to indicate the partial radiative width for a transition leading to a specific bound state.

A nice example of the onset of the resonance and interference terms in the neutron capture cross section is provided by the ${ }^{16} \mathrm{O}(n, \gamma)^{17} \mathrm{O}$ reaction for energies up to about $1 \mathrm{MeV}$. Here, the well known $J^{\pi}=3 / 2^{-} p$-wave resonance at $434 \mathrm{KeV}$, interferes with the smooth DRC $p$-wave component as shown in Figure 1. Both the capture transitions leading to the $J^{\pi}=5 / 2^{+}$ground state and to the $J^{\pi}=1 / 2^{+}$ first excited state in ${ }^{17} \mathrm{O}$ show typical interference patterns as a function of the incident neutron energy. The calculation of the DRC component was done using the $1 d_{5 / 2}$ and $2 s_{1 / 2}$ single-particle wave functions calculated from a Woods-Saxon potential with standard geometrical parameters $r_{0}=1.236 \mathrm{fm}, d=0.62 \mathrm{fm}$ and $V_{s o}=7.5 \mathrm{MeV}$. The well depth was adjusted to reproduce the experimental binding energies of the two states $\left(V_{0}=52.9\right.$ and $V_{0}=54.5$ respectively for the ground and first excited state). The spectroscopic factors for both the bound states were taken to be unity. The wave functions for the continuum were calculated using the same potential parameters with a well-depth of $V_{0}=54.5 \mathrm{MeV}$. With a resonance energy $E_{r}=434 \mathrm{KeV}$ and neutron width $\Gamma_{n}=45 \mathrm{KeV}$, the partial gamma-ray widths necessary to reproduce the experimental data were $\Gamma_{\gamma_{0}}=\Gamma_{\gamma_{1}}=0.5 \mathrm{eV}$. No other adjustable parameters were used in the calculation. The obtained total radiative width was therefore $\Gamma_{\gamma}=1.0 \mathrm{eV}, 40 \%$ smaller than the BNL compiled value [3]. 


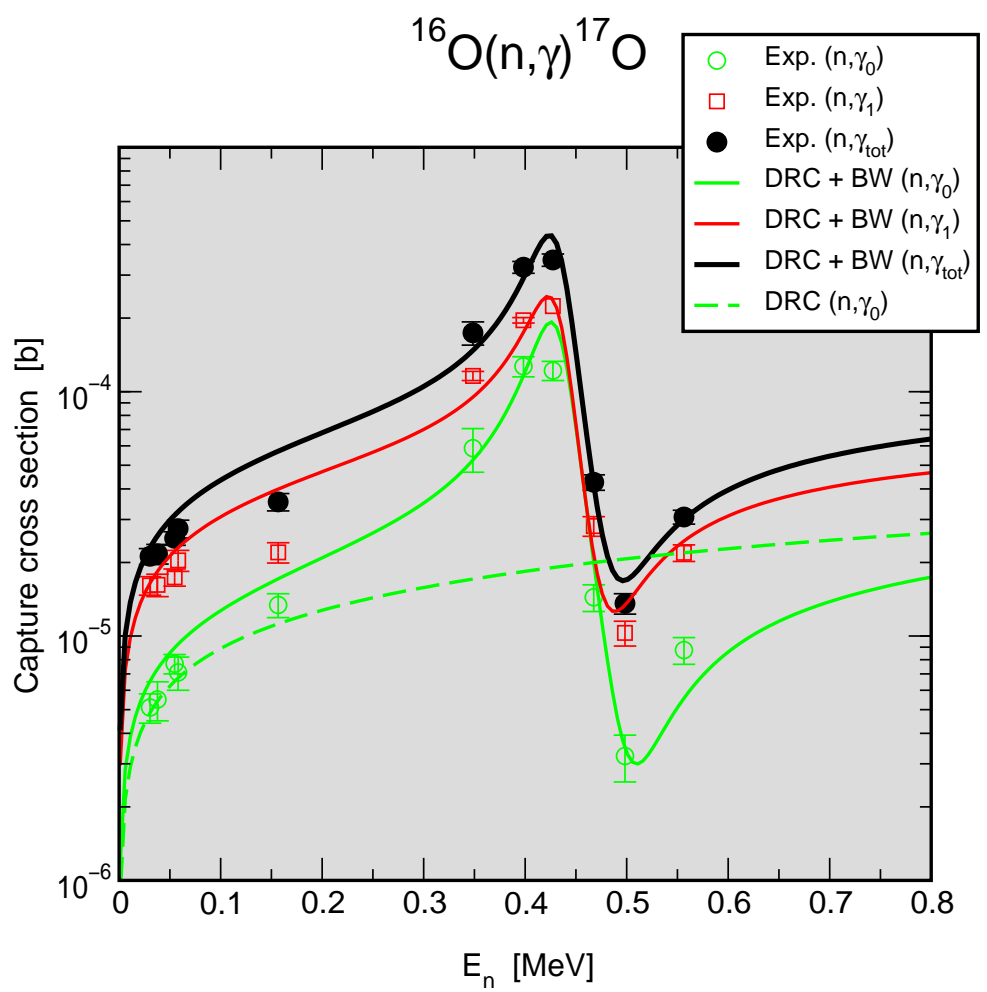

FIGURE 1. ${ }^{16} \mathrm{O}(n, \gamma){ }^{17} \mathrm{O}$ cross section for neutron energies around the $434 \mathrm{KeV}$ neutron resonance. The experimental values are from [4]. $\left(n, \gamma_{0}\right)$ and $\left(n, \gamma_{1}\right)$ indicates the transition leading to the ground and first excited state in ${ }^{17} \mathrm{O}$, respectively.

\section{PHOTO DISSOCIATION}

The time-reversal invariance of nuclear reactions provides a relation between the capture and photo-neutron cross section (detailed balance). In fact, once we define the reaction as

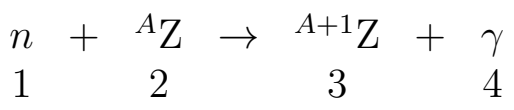

the relation connecting the direct and inverse processes is simply given by

$$
\sigma_{\gamma, n}=\frac{k_{n}^{2}}{k_{\gamma}^{2}} \frac{2 J_{2}+1}{2 J_{3}+1} \sigma_{n, \gamma}
$$

where $k_{n}$ is the wavenumber of the $n+{ }^{A} \mathrm{Z}$ relative motion.

An interesting example of photo-dissociation process is given by the $\gamma+{ }^{9} \mathrm{Be} \rightarrow$ ${ }^{8} \mathrm{Be}+n$ reaction. In fact, its time-reversal process, the neutron capture by ${ }^{8} \mathrm{Be}$, is supposed to play a crucial role in the so-called $\alpha$-induced recombination process during the post-collapse phase of a TypeII supernova [5]. The neutron capture reaction rate cannot be measured directly because of the $\alpha$-decay of ${ }^{8} \mathrm{Be}$. However, the photo-dissociation experiment can be performed and, indeed, some data are 


$$
{ }^{9} \mathrm{Be}+\gamma \rightarrow{ }^{8} \mathrm{Be}+\mathrm{n}
$$

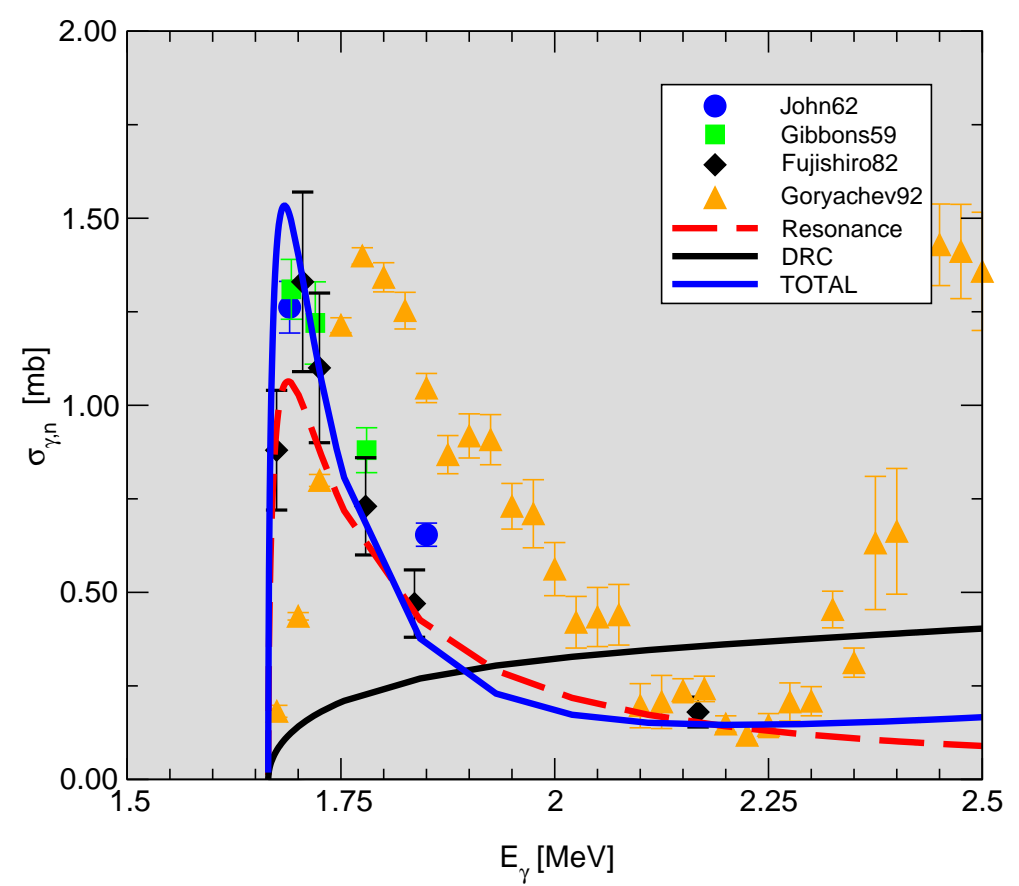

FIGURE 2. Photo dissociation of ${ }^{9} \mathrm{Be}$ in the vicinity of the ${ }^{8} \mathrm{Be}+n$ emission threshold. Experimental data are from the EXFOR compilation [6] (see there for references). The DRC, resonance and interference terms are calculated as described in the text.

available. From the nuclear structure point of view, this is an interesting case as the first excited $J^{\pi}=1 / 2^{+}$state of ${ }^{9} \mathrm{Be}$, is located just above the neutron separation threshold. In view of the discussion we made above concerning the interference between the DRC and the resonance process, some interesting behavior of the photo-neutron cross section close to threshold can be expected. The structure properties of the $J^{\pi}=1 / 2^{+}$state are also still quite uncertain. Its most obvious property is that it is located below the $J^{\pi}=5 / 2^{+}$state. The lowering of the $2 s_{1 / 2}$ orbit in light systems (of which the $J^{\pi}=1 / 2^{+}$state is supposed to be mainly composed) is the subject or recent interesting investigations.

We have performed the calculation of the ${ }^{9} \mathrm{Be}(\gamma, n){ }^{8} \mathrm{Be}$ cross section using a DRC model for the bound-to-continuum transition plus resonance and interference contributions. The results of this calculation is shown in Figure 2. The parameters and the technique used for the calculation of the DRC component are similar to those employed for the calculation of the neutron capture cross section discussed above. Here, however, the ${ }^{9} \mathrm{Be}$ ground state has a configuration of type $\left|{ }^{8} \mathrm{Be}\left(0^{+}\right) \times \nu\left(1 p_{3 / 2}\right) ; 3 / 2^{-}\right\rangle$. In addition, in this case we made a shell model calculation using the Cohen-Kurath residual interaction for the p-shell model space and obtained $S=0.5$. The parameters of the resonance state were taken from the work of Barker [7]. These were $E_{r}=73.4 \mathrm{KeV}, \Gamma_{n}=255 \mathrm{KeV}$ and $\Gamma_{\gamma}=0.53 \mathrm{eV}$. 


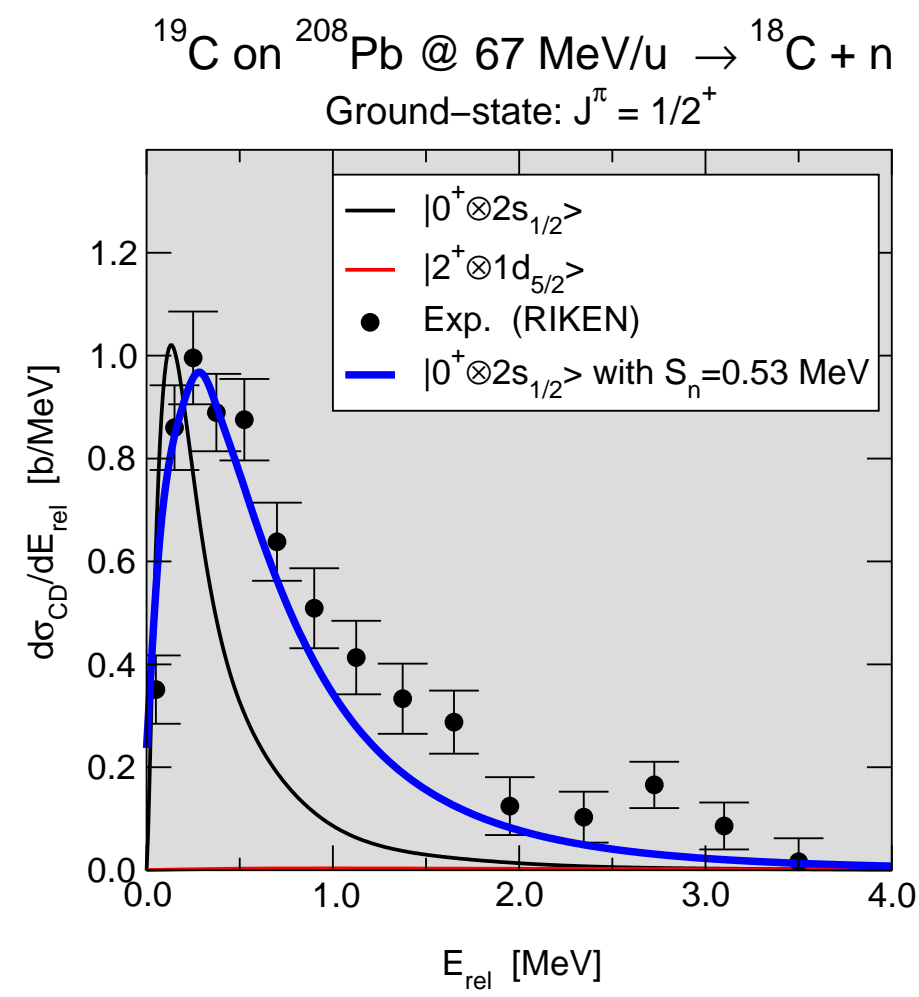

FIGURE 3. Coulomb dissociation cross section spectrum for ${ }^{19} \mathrm{C} \rightarrow{ }^{18} \mathrm{C}+n$ at $67 \mathrm{~A} \mathrm{MeV}$ incident energy as a function of the ${ }^{18} \mathrm{C}-n$ relative energy. The experimental data are from a recent RIKEN experiment [9]. The solid thin line shows the cross section as derived assuming a neutron separation energy of $160 \mathrm{KeV} .\left|{ }^{18} \mathrm{C}\left(2^{+}\right) \times \nu\left(1 d_{5 / 2}\right) ; 1 / 2^{+}\right\rangle$configuration contribution is negligible. The main configuration is the $\left|{ }^{18} \mathrm{C}\left(0^{+}\right) \times \nu\left(2 s_{1 / 2}\right) ; 1 / 2^{+}\right\rangle$with a spectroscopic factor of $S=0.67$. See [9] for details.

From the results shown in Figure 2 we can see that the peak observed just above threshold is due to the presence of the $J^{\pi}=1 / 2^{+}$state. The DRC component is rather smooth and an interference effect seems to take place reducing the cross section at $\epsilon_{\gamma} \approx 2 \mathrm{MeV}$ below the DRC component.

\section{COULOMB DISSOCIATION}

If a reaction is induced by real photons, the above equation can be employed directly to relate the direct and inverse channel. If, however, the photo-neutron process is to be induced by the virtual photons provided by a heavy- $Z$ target, like in for heavy-ion collisions, the process is more complicated.

In the semi-classical limit (Coulomb trajectories) the cross section distribution for Coulomb dissociation is related to the photo-nuclear cross section by the relation

$$
\frac{d \sigma_{c d}^{\mathrm{EL}}}{d E_{x}}=\int 2 \pi b d b \frac{N_{\mathrm{EL}}\left(E_{i}, E_{x}, b\right)}{E_{x}} \sigma_{\gamma, n}^{\mathrm{EL}}=\frac{N_{\mathrm{EL}}\left(E_{i}, E_{x}\right)}{E_{x}} \sigma_{\gamma, n}^{\mathrm{EL}}
$$


where $E_{x}=S_{n}+E_{r e l}$ is the excitation energy $\left(E_{r e l}\right.$ is the ${ }^{18} \mathrm{C}-n$ relative energy) and $N_{\text {EL }}$ the virtual-photon number of multipolarity EL at given incident energy $E_{i}$ and impact parameter $b$. Analytical expression for EL can be worked out and can be found in the literature [8].

Many breakup experiments have been recently performed using incident radioactive ion beams. Examples includes ${ }^{8} \mathrm{~B} \rightarrow{ }^{7} \mathrm{Be}+p,{ }^{11} \mathrm{Li} \rightarrow{ }^{9} \mathrm{Li}+2 n,{ }^{11} \mathrm{Be} \rightarrow{ }^{10} \mathrm{Be}+n$, ${ }^{14} \mathrm{Be} \rightarrow{ }^{12} \mathrm{Be}+2 n$ and others. We report here on the results recently obtained in RIKEN of the breakup of ${ }^{19} \mathrm{C}$, the heaviest of these loosely bound systems observed so far. This case is interesting as the structure of ${ }^{19} \mathrm{C}$ was poorly known. The spin and parity of the ground state was uncertain as well as its neutron separation energy. The details of the experiment can be found in the reference [9]. Here we show the experimental results together with our calculation in Figure 3. The experiment was performed at $E_{i}=67 \mathrm{~A} \mathrm{MeV}$. From a comparison of the experimental data with the theoretical calculation it has been concluded that: 1) the spin and parity of the ${ }^{19} \mathrm{C}$ ground state is $\left.1 / 2^{+}, 2\right)$ the neutron binding energy is $S_{n}=0.53 \pm 0.13 \mathrm{MeV}$. Here we limit ourselves in observing that the Coulomb dissociation spectrum is well

reproduced by the calculation based on a direct breakup model in which the ${ }^{19} \mathrm{C}$ ground state contains a large fraction $(S=0.67)$ of the $\left|{ }^{18} \mathrm{C}\left(0^{+}\right) \times \nu\left(2 s_{1 / 2}\right) ; 1 / 2^{+}\right\rangle$ configuration.

\section{ACKNOWLEDGMENTS}

This work has been partially supported by the European Union through its Science and Technology Programme in Japan. One of us (A.M.) would like to express his gratitude to the Department for Physics of the University of Tokyo and to RIKEN - Radiation Laboratory, for the kind hospitality offered during the period in which the most part of this work has been made. We have benefited from extensive and fruitful discussion with M. Ishihara, T. Nakamura and Y. Nagai.

\section{REFERENCES}

1. Brussaard P.J., and Glaudemans P.W.M., Shell-model applications in nuclear spectroscopy, Amsterdam: North-Holland, 1977, ch. 4, p. 64.

2. Mengoni A., Otsuka T., and Ishihara M., Phys. Rev. C 52, R2334 (1995).

3. Mughabghab S.F., Divadeenam M., and Holden N.E., Neutron Cross Sections, New York: Academic Press, 1981, vol. 1 (Part A).

4. Nagai Y, private communication (1999).

5. Görres J.,et al., Phys. Rev. C 52, 2231 (1995).

6. AA.VV. (1999), Experimental Nuclear Reaction Data File, See the web page: http://www.nndc.bnl.gov/nndc/exfor/.

7. Barker F.C., Can. J. Phys. 61, 1371 (1983).

8. Bertulani C.A., and Baur C., Phys. Rep. 163, 299 (1988).

9. Nakamura T., et al., Phys. Rev. Lett. 83, 1112 (1999). 